JOURNAL OF THE

CHUNGCHEONG MATHEMATICAL SOCIETY

Volume 26, No. 4, November 2013

http://dx.doi.org/10.14403/jcms.2013.26.4.821

\title{
ROBUSTLY CHAIN TRANSITIVE SETS WITH SHADOWING
}

\author{
Manseob LeE* And Junmi ParK**
}

\begin{abstract}
Let $f$ be a diffeomorphism of a closed $C^{\infty}$ manifold $M$, and let $\Lambda \subset M$ be a closed $f$-invariant set. We show that if $\left.f\right|_{\Lambda}$ is robustly chain transitive with shadowing, then $\Lambda$ is the hyperbolic homoclinic class.
\end{abstract}

\section{Introduction}

In differentiable dynamical systems, robustly property is a very interesting topics. For instance, Mañé [8] proved that if a diffeomorphism on two-dimensional $C^{\infty}$ manifolds is robustly transitive, then it is hyperbolic, and Díaz et al [5] proved that if a diffeomorphism on threedimensional $C^{\infty}$ manifold is robustly transitive then it is partially hyperbolic. And, in [1], the authors proved that for any dimensional $C^{\infty}$ manifolds, if a diffeomorphism is robustly transitive, then it admits a dominated splitting. In this paper, we study robustly chain transitive sets for a diffeomorphism. It is weaker notion of the robustly transitivity. In fact, Mañé [7] showed that there exists a diffeomorphism $f$ on the three dimensional torus $\mathbb{T}^{3}$ satisfies for the diffeomorphism $f \in \operatorname{Diff}\left(\mathbb{T}^{3}\right)$, there is a $C^{1}$-neighbourhood $\mathcal{U}(f)$ of $f$ such that every $g \in \mathcal{U}(f)$ is transitive, but not Anosov. Actually, there are many examples for the robustly chain transitivity $[2,3,11]$. Recently, Lee in [6] has proved that if a closed invariant set is robustly chain transitive and it has $C^{1}$-robustly a kind of the shadowing property, then the set is hyperbolic. Actually,

Received August 30, 2013; Accepted October 28, 2013.

2010 Mathematics Subject Classification: Primary 37C20, 37C50.

Key words and phrases: shadowing, chain transitive, transitive, hyperbolic.

Correspondence should be addressed to Manseob Lee, lmsds@mokwon.ac.kr.

*This work is supported by Basic Science Research Program through the National Research Foundation of Korea(NRF) funded by the Ministry of Education, Science and Technology (No. 2011-0007649).

**This work is supported by BK21 math vision 2020project by the National Research Foundation of Korea(NRF). 
Lee showed in [6] that a robustly chain transitive diffeomorphism with orbital shadowing is hyperbolic. Here, the orbital shadowing property is a general notion of the shadowing property. However, still we don't know that if a diffeomorphism has the orbital shadowing property on the robustly chain transitive set then it is the homoclinic class. In this paper we study that if a diffeomorphism has the shadowing property on the robustly chain transitive set then it is the hyperbolic homoclinic class.

Let $M$ be a closed $C^{\infty}$ manifold, and let $\operatorname{Diff}(M)$ be the space of diffeomorphisms of $M$ endowed with the $C^{1}$-topology. Denote by $d$ the distance on $M$ induced from a Riemannian metric $\|\cdot\|$ on the tangent bundle $T M$. Let $f \in \operatorname{Diff}(M)$ and $\Lambda$ be a closed $f$-invariant set.

The set $\Lambda$ is transitive if there is a point $x \in \Lambda$ such that $\omega(x)=\Lambda$. Here $\omega(x)$ is the forward limit set of $x$. For given $x, y \in M$, we write $x \rightsquigarrow y$ if for any $\delta>0$, there is a finite $\delta$-pseudo orbit $\left\{x_{i}\right\}_{i=0}^{n}(n \geq 1)$ of $f$ such that $x_{0}=x$ and $x_{n}=y$. For any $x, y \in \Lambda$, we write $x \rightsquigarrow \Lambda y$ if $x \rightsquigarrow y$ and $\left\{x_{i}\right\}_{i=0}^{n} \subset \Lambda(n \geq 1)$. We say that the set $\mathcal{C}(f)$ is chain transitive if for any $x, y \in \mathcal{C}(f), x \rightsquigarrow \mathcal{C}(f) y$. Note that by the definition, a transitive set is a chain transitive set, but the converse is not true (see [4, Example 1.5]). The notion of the chain transitive sets is well studied by [4], and $C^{1}$-generically, the chain transitive set is the limit for the Hausdorff metric of a sequence of periodic orbits(see [4, Theorem 4]).

Denote by $\left.f\right|_{\Lambda}$ the restriction of $f$ to the set $\Lambda$. Let $U \subset M$ be a compact neighborhood $\Lambda$, and put $\Lambda_{f}(U)=\bigcap_{n \in \mathbb{Z}} f^{n}(U)$. A set $\Lambda$ is locally maximal in $U$ if there is a compact neighborhood $U$ of $\Lambda$ such that $\Lambda=\Lambda_{f}(U)$.

We say the set $\Lambda$ is robustly transitive (or, $\left.f\right|_{\Lambda}$ is robustly transitive) if (i) there are a neighborhood $U$ of $\Lambda$ and a $C^{1}$-neighborhood $\mathcal{U}(f)$ of $f$ such that $\Lambda_{f}(U)=\Lambda=\bigcap_{n \in \mathbb{Z}} f^{n}(U)$ (that is, $\Lambda$ is locally maximal) (ii) for any $g \in \mathcal{U}(f), \Lambda_{g}(U)=\bigcap_{n \in \mathbb{Z}} g^{n}(U)$ is transitive for $g$. A diffeomorphism $f \in \operatorname{Diff}(M)$ is transitive if there is a point $x \in M$ such that $\omega(x)=M$. In this paper, we consider the chain transitive set in a robust way. Now, we introduce the notion of the robustly chain transitive set.

Definition 1.1. Let $f \in \operatorname{Diff}(M)$. We say the set $\mathcal{C}(f)$ is robustly chain transitive (or, $\left.f\right|_{\mathcal{C}(f)}$ is robustly chain transitive) if

(i) there is a neighborhood $U$ of $\mathcal{C}(f)$ and a $C^{1}$-neighborhood $\mathcal{U}(f)$ of $f$ such that $\Lambda_{f}(U)=\mathcal{C}(f)=\bigcap_{n \in \mathbb{Z}} f^{n}(U)$ (that is, $\Lambda$ is locally maximal)

(ii) for any $g \in \mathcal{U}(f), \Lambda_{g}(U)=\bigcap_{n \in \mathbb{Z}} g^{n}(U)$ is chain transitive for $g$. 
For $\delta>0$, a sequence of points $\left\{x_{i}\right\}_{i=a}^{b}(-\infty \leq a<b \leq \infty)$ in $M$ is called a $\delta$-pseudo orbit of $f$ if $d\left(f\left(x_{i}\right), x_{i+1}\right)<\delta$ for all $a \leq i \leq b-1$. For given $x, y \in M$, we write $x \rightsquigarrow y$ if for any $\delta>0$, there is a $\delta$-pseudo orbit $\left\{x_{i}\right\}_{i=a}^{b}(a<b)$ of $f$ such that $x_{a}=x$ and $x_{b}=y$. We say that $f$ has the shadowing property on $\Lambda$ if for every $\epsilon>0$ there is $\delta>0$ such that for any $\delta$-pseudo orbit $\left\{x_{i}\right\}_{i=a}^{b} \subset \Lambda$ of $f(-\infty \leq a<b \leq \infty)$, there is a point $y \in M$ such that $d\left(f^{i}(y), x_{i}\right)<\epsilon$ for all $a \leq i \leq b-1$.

The shadowing property usually plays an important role in the investigation of stability theory and ergodic theory. For instance, in $[9,10]$, the authors showed that every $f$ satisfying both Axiom A and the strong transversality condition has the shadowing property. Since such a system is structurally stable, there is a $C^{1}$-neighborhood $\mathcal{U}(f)$ of $f$ such that every $g \in \mathcal{U}(f)$ has the shadowing property. Also, Sakai [12] proved that if there is a $C^{1}$-neighborhood $\mathcal{U}(f)$ of $f$, for any $g \in \mathcal{U}(f) g$ has the shadowing property, then $f$ satisfies both Axiom $\mathrm{A}$ and the strong transversality condition.

We say that $\Lambda$ is hyperbolic if the tangent bundle $T_{\Lambda} M$ has a $D f$ invariant splitting $E^{s} \oplus E^{u}$ and there exists constants $C>0$ and $0<$ $\lambda<1$ such that

$$
\left\|\left.D_{x} f^{n}\right|_{E_{x}^{s}}\right\| \leq C \lambda^{n} \text { and }\left\|\left.D_{x} f^{-n}\right|_{E_{x}^{u}}\right\| \leq C \lambda^{n}
$$

for all $x \in \Lambda$ and $n \geq 0$.

In this paper, we may assume that $\mathcal{C}(f)$ is a nontrivial chain transitive set of $f$. Here a nontrivial set is not just one orbit. We now study the relation of robust chain transitivity, hyperbolicity and the shadowing property over an arbitrary dimension manifold.

It is well known that if $p$ is a hyperbolic periodic point of $f$ with period $k$ then the sets

$$
\begin{aligned}
& W^{s}(p)=\left\{x \in M: f^{k n}(x) \rightarrow p \quad \text { as } \quad n \rightarrow \infty\right\} \quad \text { and } \\
& W^{u}(p)=\left\{x \in M: f^{-k n}(x) \rightarrow p \quad \text { as } \quad n \rightarrow \infty\right\}
\end{aligned}
$$

are $C^{1}$-injectively immersed submanifolds of $M$.

A point $x \in W^{s}(p) \cap W^{u}(p)$ is called a homoclinic point of $f$ associated to $p$, and it is said to be a transversal homoclinic point of $f$ if the above intersection is transversal at $x$, i.e., $x \in W^{s}(p) \pitchfork W^{u}(p)$. The closure of the homoclinic points of $f$ associated to $p$ is called the nontransversal homoclinic class of $p$, and it is denoted by $\widetilde{H}_{f}(p)$. The closure of the transversal homoclinic points of $f$ associated to $p$ is called the transversal homoclinic class of $p$, and it is denoted by $H_{f}(p)$. Clearly, $H_{f}(p) \subset \widetilde{H}_{f}(p)$. The following is the main theorem in this paper. 
Theorem 1.2. Let $f \in \operatorname{Diff}(M)$. If $\left.f\right|_{\mathcal{C}(f)}$ is robustly chain transitive with shadowing, then $\mathcal{C}(f)$ is $H_{f}(p)$ and it is hyperbolic.

Note that in this paper, we say that if $\left.f\right|_{\mathcal{C}(f)}$ is robustly (chain) transitive with shadowing, then (i) $f$ has the shadowing property on $\mathcal{C}(f)$, and (ii) there are a $C^{1}$-neighborhood $\mathcal{U}(f)$ of $f$ and a compact neighborhood $U$ of $\mathcal{C}(f)$ such that for any $g \in \mathcal{U}(f), \Lambda_{g}(U)=\bigcap_{n \in \mathbb{Z}} g^{n}(U)$ is chain transitive, and $\left.g\right|_{\Lambda_{g}(U)}$ has the shadowing property.

\section{Proof of Theorem 1.2}

Let $M$ be as before, and $f \in \operatorname{Diff}(M)$. Let $P(f)$ be the set of periodic points of $f$. If $\Lambda$ is transitive set then every $p \in \Lambda \cap P(f)$ is saddle, that is, there is no eigenvalues of $D_{p} f^{\pi(p)}$ with modulus equal to 1 , at least one of them is greater than 1 , at least one of them is smaller than 1 , where $\pi(p)$ is the minimum period of $p$. Note that there are a $C^{1}$-neighborhood $\mathcal{U}(f)$ and a neighborhood $U$ of $p$ such that for all $g \in \mathcal{U}(f)$, there is a unique hyperbolic periodic point $p_{g} \in U$ of $g$ with the same period as $p$ and $\operatorname{index}\left(p_{g}\right)=\operatorname{index}(p)$. Here index $(p)=\operatorname{dim} E_{p}^{s}$, and the point $p_{g}$ is called the continuation of $p$. Thus we get the following result which due to Mañé[8] that holds in any dimensional.

TheOREM 2.1. [5] Let $\Lambda$ be a robustly transitive set. The following statements are equivalent:

(i) there is a $C^{1}$-neighborhood $\mathcal{U}(f)$ of $f$ such that for any $g \in \mathcal{U}(f)$, any periodic points of $\Lambda_{g}(U)=\bigcap_{n \in \mathbb{Z}} g^{n}(U)$ are hyperbolic and have the same index;

(ii) there is a $C^{1}$-neighborhood $\mathcal{U}(f)$ of $f$ such that for any $g \in \mathcal{U}(f)$, $\Lambda_{g}(U)$ is hyperbolic.

REMARK 2.2. Let $\Lambda$ be a nontrival transitive set. Then there are a sequence $\left\{f_{n}\right\}$ and a periodic orbits $P_{n}$ of $f_{n}$ with period $\pi\left(P_{n}\right) \rightarrow \infty$ such that $P_{n} \rightarrow \Lambda$ and $f_{n} \rightarrow f$ as $n \rightarrow \infty$ (see [16, Corollary 2.7.1]). If $\Lambda$ is locally maximal, then there is a periodic point in $\Lambda$.

To prove Theorem 1.2, it is enough to show the following propositions by Theorem 2.1 .

Proposition 2.3. Let $\mathcal{U}(f)$ and $U$ be as in the definition, and let $\left.f\right|_{\Lambda}$ be robustly transitive with shadowing. Then for any hyperbolic periodic points $p, q \in \Lambda_{g}(U) \cap P(g)(g \in \mathcal{U}(f))$, index $(p)=\operatorname{index}(q)$.

To prove Proposition 2.3, we need the following lemmas. 
Lemma 2.4. Let $\left.f\right|_{\Lambda}$ be transitive, and let $p, q \in \Lambda$ be hyperbolic periodic points of $f$. Suppose that $f$ has the shadowing property on $\Lambda$. Then

$$
W^{s}(p) \cap W^{u}(q) \neq \emptyset \quad \text { and } \quad W^{u}(p) \cap W^{s}(q) \neq \emptyset .
$$

Proof. Let $p, q \in \Lambda$ are hyperbolic periodic points of $f$, and let $\epsilon(p)>$ 0 and $\epsilon(q)>0$ be as before with respect to $p$ and $q$. For simplicity, we may assume that $f(p)=p$ and $f(q)=q$. Take $\epsilon=\min \{\epsilon(p), \epsilon(q)\}$. Let $0<\delta<\epsilon$ be the number of the shadowing property of $\left.f\right|_{\Lambda}$ for $\epsilon$. Since $\left.f\right|_{\Lambda}$ is transitive, there exists $x \in \Lambda$ such that $\omega(x)=\Lambda$. Then there exist $l_{1}>0$ and $l_{2}>0$ such that $d\left(f^{l_{1}}(x), p\right)<\delta$, and $d\left(f^{l_{2}}(x), q\right)<$ $\delta$. We may assume that $l_{2}>l_{1}$. Then we get a finite $\delta$-pseudo orbit $\left\{p, f^{l_{1}}(x), f^{l_{1}+1}(x), \ldots, f^{l_{2}-1}(x), q\right\}$. Now we construct a $\delta$-pseudo orbit as follows: Put (i) $l_{2}=l_{1}+k$ for some $k>0$, (ii) $f^{i}(p)=x_{i}$ for $i \leq 0$, (iii) $f^{l_{1}+i}(x)=x_{i}$ for $1 \leq i \leq k-1$, and (iv) $f^{l_{2}+i}(q)=x_{k+i}$ for $i \geq 0$. Then

$$
\begin{aligned}
\xi & =\left\{\ldots, p, x_{0}(=p), f^{l_{1}}(x), f^{l_{1}+1}(x), \ldots, f^{l_{1}+k-1}(x), f^{l_{2}}(x)(=q), q, \ldots\right\} \\
& =\left\{\ldots, x_{-2}, x_{-1}, x_{0}(=p), x_{1}, x_{2}, \ldots, x_{k-1}, x_{k}(=q), x_{k+1}, \ldots,\right\} .
\end{aligned}
$$

It is clear that $\xi \subset \Lambda$. By the shadowing property of $\left.f\right|_{\Lambda}$, we can find a point $z \in M$ such that $d\left(f^{i}(z), x_{i}\right)<\epsilon$ for all $i \in \mathbb{Z}$. Thus

$$
d\left(f^{i}(z), x_{i}\right)=d\left(f^{i}(z), p\right)<\epsilon
$$

for all $i \leq 0$ and

$$
d\left(f^{k+i}(z), x_{k+i}\right)=d\left(f^{k+i}(z), q\right)<\epsilon
$$

for all $i \geq 0$. Then one can see that $z \in W_{\epsilon}^{u}(p)$, and $f^{k}(z) \in W_{\epsilon}^{s}(q)$, where $W_{\epsilon}^{s}(q), W_{\epsilon}^{u}(p)$ are as usual. Thus, $z \in W^{u}(p) \cap W^{s}(q) \neq \emptyset$. Other cases is similar.

The following, so-called Franks' lemma, will play essential roles in our proof.

Lemma 2.5. Let $\mathcal{U}(f)$ be any given $C^{1}$-neighborhood of $f$. Then there exists $\epsilon>0$ and a $C^{1}$-neighborhood $\mathcal{U}_{0}(f) \subset \mathcal{U}(f)$ of $f$ such that for given $g \in \mathcal{U}_{0}(f)$, a finite set $\left\{x_{1}, x_{2}, \ldots, x_{N}\right\}$, a neighborhood $U$ of $\left\{x_{1}, x_{2}, \ldots, x_{N}\right\}$ and linear maps $L_{i}: T_{x_{i}} M \rightarrow T_{g\left(x_{i}\right)} M$ satisfying $\left\|L_{i}-D_{x_{i}} g\right\| \leq \epsilon$ for all $1 \leq i \leq N$, there exists $g^{\prime} \in \mathcal{U}(f)$ such that $g^{\prime}(x)=g(x)$ if $x \in\left\{x_{1}, x_{2}, \ldots, x_{N}\right\} \cup(M \backslash U)$ and $D_{x_{i}} g^{\prime}=L_{i}$ for all $1 \leq i \leq N$. 
Remark 2.6. A diffeomorphism $f$ said to be Kupka-Smale if the periodic points of $f$ are hyperbolic and thier invariant manifolds are transverse intersections. It is well-known that the set of Kupka-Smale diffeomorphisms is $C^{1}$-residual in $\operatorname{Diff}(M)$.

Proof of Proposition 2.3. Let $\left.f\right|_{\Lambda}$ be robustly transitive with shadowing, and let $\mathcal{U}(f)$ be as in the definition. For any $g \in \mathcal{U}(f)$, let $p, q \in \Lambda_{g}(U) \cap P(g)$ be hyperbolic periodic points. By Lemma 2.5, there is a $C^{1}$-neighborhood $\mathcal{U}_{0}(g) \subset \mathcal{U}(f)$ of $g$, such that for any $h \in \mathcal{U}_{0}(g)$, there are the continuations $p_{h}$ and $q_{h}($ of $p$ and $q)$ in $\Lambda_{h}(U) \cap P(h)$. Since $\left.f\right|_{\Lambda}$ is robustly transitive, for the neighborhood $U, \Lambda \subset \operatorname{int} U$, we may assume that $\Lambda_{h}(U) \subset \operatorname{int} U$ for any $g \in \mathcal{U}(f)$ reducing $\mathcal{U}(f)$ if necessary. To derive a contradiction, $\operatorname{suppose}$ that index $(p)<\operatorname{index}(q)$, and thus $\operatorname{dim} W^{s}\left(p_{g}\right)+\operatorname{dim} W^{u}\left(q_{g}\right)<\operatorname{dim} M$ (the other case is similar). Here $W^{s}\left(p_{g}\right)$ and $W^{u}\left(p_{g}\right)$ are the stable and the unstable manifolds of $p_{g}$ and $q_{g}$ with respect to $g$.

Since $\left.f\right|_{\Lambda}$ is robustly transitive, we can take a Kupka-Smale diffeomorphism $h \in \mathcal{U}_{0}(g)$. Since $\operatorname{dim} W^{s}\left(p_{g}\right)=\operatorname{dim} W^{s}\left(p_{h}\right)$ and $\operatorname{dim} W^{u}\left(q_{g}\right)=$ $\operatorname{dim} W^{u}\left(q_{h}\right)$,

$$
W^{s}\left(p_{h}\right) \cap W^{u}\left(q_{h}\right)=\emptyset .
$$

By Lemma 2.4, this is a contradiction.

Proposition 2.7. Let $\mathcal{U}(f)$ and $U$ be as in the definition, and let $\left.f\right|_{\Lambda}$ be robustly transitive with shadowing. For any $g \in \mathcal{U}(f)$, every periodic point of $\Lambda_{g}(U)$ is hyperbolic.

Let $\Lambda$ be a closed $f$-invariant subset of $M$.

Lemma 2.8. [15, Lemma 2.4] Let $\Lambda$ be locally maximal in $U$, and let $\mathcal{U}(f)$ be given. If $p \in \Lambda_{g}(U) \cap P(g)(g \in \mathcal{U}(f))$ is not hyperbolic, then there is $g_{1} \in \mathcal{U}(f)$ possessing hyperbolic periodic points $q_{1}$ and $q_{2}$ in $\Lambda_{g_{1}}(U)$ with different indices.

Proof of Proposition 2.7. Let $\left.f\right|_{\Lambda}$ be robustly transitive with shadowing. Then there exist a $C^{1}$-neighborhood $\mathcal{U}(f)$ of $f$ and a compact neighborhood $U$ of $\Lambda$ such that for any $g \in \mathcal{U}(f), \Lambda_{g}(U)=\bigcap_{n \in \mathbb{Z}} g^{n}(U)$ is transitive and $g$ has the shadowing property on $\Lambda_{g}(U)$. For a contradiction, suppose that there is a non hyperbolic point $p \in \Lambda_{g}(U) \cap P(g)$ for some $g \in \mathcal{U}(f)$. By Franks' Lemma 2.5 and Lemma 2.8, there is $g_{1} \in$ $\mathcal{U}_{0}(g) \subset \mathcal{U}(f)$ possessing hyperbolic periodic points $q_{1}$ and $q_{2}$ in $\Lambda_{g_{1}}(U)$ with different indices, that is, $\operatorname{index}\left(q_{1}\right) \neq \operatorname{index}\left(q_{2}\right)$. This is a contradiction by Proposition 2.3. Therefore, every $p \in \Lambda_{g}(U) \cap P(g)(g \in \mathcal{U}(f))$ 
is hyperbolic

Let $\Lambda$ be a closed $f$-invariant set. Recall that $\left.f\right|_{\Lambda}$ is transitive if there is a point $x \in \Lambda$ such that $\omega(x)=\Lambda$. Also, we see that for any non-empty open sets $U, V$ of $\Lambda$, if there is $n>0$ such that $f^{n}(U) \cap V \neq \emptyset$, then $\left.f\right|_{\Lambda}$ is transitive. Then two notions are equivalent. The following lemmas were proved by Sakai [14, Lemma 2.3, and Lemma 2.4 ].

Lemma 2.9. Let $\Lambda$ be a closed $f$-invariant set, and let $U$ be a locally maximal neighborhood of $\Lambda$. If $f$ has the shadowing property on $\Lambda$, then the shadowing point can be taken from $\Lambda$.

Lemma 2.10. If $\left.f\right|_{\mathcal{C}(f)}$ is locally maximal chain transitive with shadowing, then $\left.f\right|_{\mathcal{C}(f)}$ is transitive.

From the Lemma 2.10 , if $\left.f\right|_{\mathcal{C}(f)}$ is robustly chain transitive with the shadowing then $\left.f\right|_{\mathcal{C}(f)}$ is robustly transitive.

Recall that chain transitive sets are characterized as all compact invariant sets which are the Hausdorff limit of a sequence of periodic orbits under the $C^{1}$-generic assumption (see [4, Theorem 4]).

Lemma 2.11. Let $\Lambda$ be a transitive set and locally maximal in $U$. If $\left.f\right|_{\Lambda}$ is robustly transitive with shadowing then $\Lambda=H_{f}(p)$.

Proof. Suppose that $\left.f\right|_{\Lambda}$ is robustly transitive with shadowing. Since $\Lambda$ is a transitive set and locally maximal, by Pugh's closing lemma, there is a hyperbolic periodic point $p \in \Lambda$. For simplicity, we may assume that $f(p)=p$. Since $p$ is hyperbolic, there is $\epsilon(p)>0$ such that for any $x \in \Lambda$ if $d\left(f^{i}(x), p\right)<\epsilon(p)$ for all $i \leq 0$ then $x \in W_{\epsilon(p)}^{u}(p)$ and if $d\left(f^{i}(x), p\right)<\epsilon(p)$ for all $i \geq 0$ then $x \in W_{\epsilon(p)}^{s}(p)$. Take $\epsilon=\epsilon(p) / 4$, and let $0<\delta<\epsilon$ be a number of the shadowing property of $\left.f\right|_{\Lambda}$. Since $\Lambda$ is a transitive set, there is $x \in \Lambda$ such that $\omega(x)=\Lambda$. Thus there are $k_{1}>0$ and $k_{2}>0$ such that $d\left(f^{k_{1}}(x), p\right)<\delta / 4$, and $d\left(f^{k_{2}}(x), x\right)<\delta / 4$. Then we construct a $\delta$-pseudo orbit

$$
\begin{aligned}
\left\{x_{i}: i \in \mathbb{Z}\right\}=\{ & \ldots, f^{-1}(x), x, f(x), \ldots, f^{k_{1}-1}(x), p, f^{k_{1}+1}(x), f^{k_{1}+2}(x), \\
& \left.\ldots, f^{k_{2}-1}(x), x, f(x), f^{2}(x), \ldots,\right\} \\
=\{ & \left\{, x_{-k_{1}}, x_{-k+1}, \ldots, x_{-1}, p\left(=x_{0}\right), x_{1}, \ldots, x_{k_{2}}, \ldots\right\} .
\end{aligned}
$$

By the shadowing property, there is $y \in B_{\epsilon}(p) \cap \Lambda$ such that $d\left(f^{i}(x), x_{i}\right)<$ $\epsilon$ for all $i \in \mathbb{Z}$. Thus $y \in B_{\epsilon}(p) \cap W^{s}(p) \cap W^{u}(p)$, and so $y \in \widetilde{H}_{f}(p)$. Since 
$\left.f\right|_{\Lambda}$ is robustly transitive with shadowing, as in the proof of [13, Proposition 3.1], we know $y \in B_{\epsilon}(p) \cap W^{s}(p) \pitchfork W^{u}(p)$. Thus $\Lambda=H_{f}(p)$.

Proof of Theorem 1.2. Suppose that $\left.f\right|_{\mathcal{C}(f)}$ is robustly chain transitive with shadowing. There are a $C^{1}$-neighborhood $\mathcal{U}(f)$ of $f$ and a compact neighborhood $U$ of $\mathcal{C}(f)$ such that for any $g \in \mathcal{U}(f), \Lambda_{g}(U)=$ $\bigcap_{n \in \mathbb{Z}} g^{n}(U)$ is chain transitive and $g$ has the shadowing property on $\Lambda_{g}(U)$. By Lemma 2.10, $\left.f\right|_{\mathcal{C}(f)}$ is transitive and for $g \in \mathcal{U}(f),\left.g\right|_{\Lambda_{g}(U)}$ is also transitive. This means that $\left.f\right|_{\mathcal{C}(f)}$ is robustly transitive. Then by Propositions 2.3 and 2.7, for any $p \in \Lambda_{g}(U) \cap P(g)$ is hyperbolic, and for any $p, q \in \Lambda_{g}(U) \cap P(g)$ then $\operatorname{index}(p)=\operatorname{index}(q)$. Thus by Theorem 2.1 and Lemma $2.11, \Lambda$ is $H_{f}(p)$ and it is hyperbolic.

\section{References}

[1] C. Bonatti, L. J. Díaz, and E. R. Pujals, A $C^{1}$-generic dichotomy for diffeomorphisms: weak forms of hyperbolicity or infinitlely many sinks or sources, Ann. of Math. 158 (2003), 355-418.

[2] C. Bonatti and L. J. Díaz, Persistence of transitive diffeomorphisms, Ann. Math. 143 (1995), 367-396.

[3] M. Carvalho, Sinai-Ruelle-Bowen measures for $N$-dimensional derived from Anosov diffeomorphisms, Ergodic Theory Dyn. Syst. 13 (1993) 21-44.

[4] C. Crovisier, Peirodic orbits and chain transitive sets of $C^{1}$ diffeomorphisms, Publ. Math. de L'iheś, 104, 87-141.

[5] L. J. Díaz, E. R. Pujals, and R. Ures, Partial hyperbolicity and robustly transitivity, Acta Math. 183 (1999), 1-43.

[6] M. Lee, Robustly chain transitive setse with orbital shadowing diffeomorphisms, Dynamical Systems, 27 (2012), 507-514.

[7] R. Mãné, Contributions to stability conjecture, Topology 17 (1978), 383-396.

[8] R. Mãné, An ergodic closing lemma, Ann. Math. 116 (1982), 503-540.

[9] S. Y. Pilyugin, "Shadowing in Dynamical Systems", Lecture Notes in Math. 1706 (Springer Verlag, Berlin, 1999).

[10] C. Robinson, Stability theorems and hyperbolicity in dynamical systems, Rocky Mountain J. Math. 7 (1977), 425-437.

[11] M. Shub, Topologically transitive diffeomorphism of $T^{4}$, Symposium on Differential Equations and Dyanmical Systems(University of Warwick, 1968/69), Lecture Notes in Mathematics, Vol. 206, Springer-Verlag, Berlin, New York, 1971, 39-40.

[12] K. Sakai, Pseudo orbit tracing property and strong transversality of diffeomorphisms on closed manifolds, Osaka J. Math. 31 (1994), 373-386.

[13] K. Sakai, $C^{1}$-stably shadowable chain components, Ergodic Theory \& Dynam. Sys. 28 (2008), 987-1029.

[14] K. Sakai, Shadowable chain transitive sets, J. Differ. Equat. Appli. 19 (2013), 1601-1618. 
[15] K. Sakai, N. Sumi, and K. Yamamoto, Diffeomorphisms satisfying the specification property, Proc. Amer. Math. Soc. 138 (2010), 315-321.

[16] D. Yang, Stably weakly shadowing transitive sets and dominated splitting. Proc. Amer. Math. Soc. 139 (2011), 2747-2751.

$*$

Department of Mathematics

Mokwon University

Daejeon 302-729, Republic of Korea

E-mail: Imsds@mokwon.ac.kr

$* *$

Department of Mathematics

Chungnam National University

Daejeon 305-764, Republic of Korea

E-mail: pjmds@cnu.ac.kr 